\title{
Pyruvate formate-lyase enables efficient growth of Escherichia coli on acetate and formate.
}

Lior Zelcbuch $^{\dagger, \ddagger}$, Steffen N. Lindner ${ }^{\circ, \ddagger}$, Yonatan Zegman ${ }^{\dagger}$, Ilya Vainberg Slutskin ${ }^{\dagger}$, Niv Antonovsky ${ }^{\dagger}$, Shmuel $^{\circ}$ Gleizer $^{\dagger}$, Ron Milo ${ }^{\dagger}$, Arren Bar-Even ${ }^{\circ, *}$

\section{Supporting Information}

\author{
Materials \& Methods
}

\section{Gene cloning}

All cloning was done using our "no background" cloning method (detailed explanation can be found here: http://openwetware.org/wiki/Milo:No background_cloning_protocol) (1). At first step the pflA and PflB gene where amplify from E. coli MG1655 genomic DNA by PCR. The sense oligos contained 6 His tag downstream to the ATG which serves as RBS insulator and also provided NsiI site for cloning the genes into pNiv (1). The antisense oligo contained a $20 \mathrm{bp}$ homologous sequence to the chloramphenicol resistance brick in order to allow the addition of this brick by overhang extension PCR. PflA was amplified using the primer pair PflA forward and PflA reverse. Since the $p f l B$ gene has a NsiI restriction site, which must not be present in the genes sequence for the cloning procedure, the site was eliminated with a silent point mutation, by using the overhang extension procedure (2). Thus, amplification of the pflB gene was carried out in two steps. First, to remove the NsiI restriction site in the gene, a PCR was performed amplifying the gene in two parts, by use of the primer pairs PflB forward and PflB A534T reverse, as well as PflB A534T forward and PflB reverse. In the second step the corresponding DNA fragments were fused by a PCR using the fragments as templates and primer pair PflB forward and PflB reverse. These PCR products were used as template for a further PCR reaction in which the addition of the chloramphenicol resistance brick accrued. This PCR product was cloned directly via blunt end ligation into pBluscript KS plasmid and screened for Chloramphenicol resistance. The plasmid then sequenced in order to validate $p f l A$ and $p f l B$ correct sequence. After The validation, $p f l A$ and $p f l B$ were restricted out of the pBluscript KS plasmid using NsiI \& XhoI restriction enzyme and ligated into pNiv plasmid (1) which contained 
RBS-C (1) upstream to the insertion point generating two plasmids pNiv RBS-C $-p f l A$ and pNiv RBS-C $-p f l B$. pNiv RBS-C - PflA plasmid was used as vector and restricted with NheI and XhoI, while pNiv RBS-C - PflB was used as insert and restricted with SpeI and SalI restriction enzymes. The ligation between pNiv RBS-C - pflA and pNiv RBS-C - pflB yielded the plasmid pNiv RBS-C - pflA - RBS-C - pflB, which has an operon containing pflA and $p f l B$ under the control of medium constitutive promoter.

\begin{tabular}{|l|l|}
\hline Name of the primer & Sequence \\
\hline PflB forward & ATGCATCATCACCATCACCACTCCGAGCTTAATGAAAAGTTAGCC \\
\hline PflB reverse & CTCTTACGTGCCCGATCAACGCTAGCTTACATAGATTGAGTGAAGGTACGAG \\
\hline PflB A534T forward & CGGTCTGCCAGATGCTTATGGCCGTGGCCGTATCA \\
\hline PflB A534T reverse & TGATACGGCCACGGCCATAAGCATCTGGCAGACCG \\
\hline PflA forward & ATGCATCATCACCATCACCACTCAGTTATTGGTCGCATTCAC \\
\hline PflA reverse & CTCTTACGTGCCCGATCAACGCTAGCTTAGAACATTACCTTATGACCGTAC \\
\hline Chloramphenicol Brick reverse & GCTAGCGTTGATCGGGCACGTAAGAG \\
\hline
\end{tabular}

\section{Gene deletion}

Gene deletions were carried out using Red/ET recombination (Quick \& Easy E. coli Gene Deletion Kit, Gene Bridge, Heidelberg, Germany), following the descriptions in the manufacturers protocol. We started by using the Keio collection strains JW3975 ( $\triangle a c e A)$ and JW0886 ( $\triangle p f l B)(3)$ and removed their kanamycin resistances by use of the flippase reaction (carried out according Quick \& Easy E. coli Gene Deletion Kit, Gene Bridge). The resulting strains were used for Red/ET recombination for construction of the double mutant, $\triangle a c e A \Delta p f B$. For PCR amplification of the kanamycin cassette with $50 \mathrm{bp}$ homologous arms for gene knock out, primer pairs pflB_KO_F and pflB_KO_R or aceA_KO_F and aceA_KO_R were used. For the verification of the integration of the kanamycin cassette and subsequently (after using flippase reaction) the removal of gene and kanamycin cassette primer pairs pflB_KO_V_F and pflB_KO_V_R or aceA_KO_V_F and aceA_KO_V_R were used.

The construction of the double mutant from both origins (i.e., deleting ace $A$ on the $\triangle p f l B$ background and deleting $p f l B$ on the $\triangle a c e A$ background) was done to exclude effects of secondary genetic differences within the initial Keio collection strains JW3975 $(\triangle a c e A)$ and JW0886 ( $\triangle p f l B)$. Growth comparisons of both double mutants revealed no differences in growth on acetate or acetate + formate, with or without further transformation with the with the $p f l B A$ overexpression plasmid. In the main text, we show and described and results for the $\triangle a c e A \triangle p f l B$ strain based on the Keio collection JW3975 ( $\triangle a c e A)$ strain.

\begin{tabular}{|l|l|l|}
\hline Name & Sequence (bold: gene flanking sequences) & Use \\
\hline pflB_KO_F & $\begin{array}{l}\text { TTTTACTGTACGATTTCAGTCAAATCTA } \\
\text { ATTACATAGATTGAGTGAAGGTAATTAA } \\
\text { CCCTCACTAAAGGGCG }\end{array}$ & $\begin{array}{l}\text { PCR amplification of kanamycin cassette for } p f l B \\
\text { deletion }\end{array}$ \\
\hline pflB_KO_R & $\begin{array}{l}\text { CGAAGTACGCAGTAAATAAAAAATCCA } \\
\text { CTTAAGAAGGTAGGTGTTACATGTAATA }\end{array}$ & $\begin{array}{l}\text { PCR amplification of kanamycin cassette for } p f l B \\
\text { deletion }\end{array}$ \\
\hline
\end{tabular}




\begin{tabular}{|l|l|l|}
\hline & CGACTCACTATAGGGCTC & \\
\hline pflB_KO_V_F & ATCGGTGGTGGTTTGTTGGT & Primer for verification of $p f l B$ deletion \\
\hline pflB_KO_V_R & ACGTCGAGTCTGTTTTGGCA & Primer for verification of $p f l B$ deletion \\
\hline aceA_KO_F & $\begin{array}{l}\text { TACCGCCTGTTAGCGTAAACCACCACAT } \\
\text { AACTATGGAGCATCTGCACATGAATTAA } \\
\text { CCCTCACTAAAGGGCG }\end{array}$ & $\begin{array}{l}\text { PCR amplification of kanamycin cassette for } \\
\text { ace } A \text { deletion }\end{array}$ \\
\hline aceA_KO_R & $\begin{array}{l}\text { GGCCTACAGTCAGCAACGGTTGTTGTT } \\
\text { GCTTAGAACTGCGATTCTTCAGTTAATA } \\
\text { CGACTCACTATAGGGCTC }\end{array}$ & $\begin{array}{l}\text { PCR amplification of kanamycin cassette for } \\
\text { ace } A \text { deletion }\end{array}$ \\
\hline aceA_KO_V_F & ACTGGGCGAAGAACGTTTCT & Primer for verification of $a c e A$ deletion \\
\hline aceA_KO_V_R & GGCATGATAAGACGCGCAAG & Primer for verification of $a c e A$ deletion \\
\hline
\end{tabular}

\section{Strain cultivation and growth experiments}

Precultures were grown aerobically at $37^{\circ} \mathrm{C}$ in $5 \mathrm{ml} \mathrm{LB}$ containing $30 \mu \mathrm{g} / \mathrm{ml}$ chloramphenicol when appropriate. For growth experiments M9 minimal medium (containing trace elements, $100 \mathrm{x}$ trace elements stock solution was prepared as follows: $498 \mathrm{mg} \mathrm{FeCl}{ }_{3}$ anhydrous, $84 \mathrm{mg} \mathrm{ZnCl}, 765 \mu \mathrm{l}$ of $0.1 \mathrm{M} \mathrm{CuCl}_{2} \cdot 2 \mathrm{H}_{2} \mathrm{O}, 210 \mu \mathrm{l}$ of $0.2 \mathrm{M}$ $\mathrm{CoCl}_{2} \cdot 6 \mathrm{H}_{2} \mathrm{O}, 1.6 \mathrm{ml}$ of $0.1 \mathrm{M} \mathrm{H}_{3} \mathrm{BO}_{3}, 8.1 \mu \mathrm{l}$ of $1 \mathrm{M} \mathrm{MnCl}_{2} \cdot 4 \mathrm{H}_{2} \mathrm{O}$, were dissolved in $1000 \mathrm{ml} \mathrm{ddH}_{2} \mathrm{O}$ containing 5 g EDTA - pH of 7.5 with $\mathrm{NaOH}$ - and sterilized by filtration threw a $0.22-\mu \mathrm{m}$ filter) was used, containing the indicated carbon sources (acetate or acetate + formate) as well as $50 \mathrm{mM} \mathrm{NaNO}$. Prior to inoculation cells were harvested by centrifugation $(4,000 \mathrm{x} \mathrm{g}, 3 \mathrm{~min}, \mathrm{RT})$, washed twice in $\mathrm{ddH}_{2} \mathrm{O}$ and inoculated in M9 media to an optical density of 0.005. Nunc 96-Well Microplates were used for cultivations (Thermo Scientific, 167008), each well contained $150 \mu 1$ culture covered with $50 \mu 1$ mineral oil (Sigma-Aldrich, M3516) to avoid evaporation. For growth experiments under anaerobic conditions, M9 media was placed for at least $24 \mathrm{~h}$ in the anaerobic chamber to allow removal of dissolved oxygen from the media.

Growth experiments were carried out in a Tecan Infinite 200 Pro plate reader (Tecan, Switzerland), placed in a vinyl anaerobic chamber $\left(\mathrm{N}_{2}\right.$ with $10 \% \mathrm{CO}_{2}, 2.5 \% \mathrm{H}_{2}$, model $\mathrm{B}$, Coy Laboratory Products). Cultivation and OD measurements in the plate reader were carried out at $37^{\circ} \mathrm{C}$. OD600 was measured in kinetic cycles consisting of $14.5 \mathrm{~min}$ without shaking, $60 \mathrm{sec}$ of $6 \mathrm{~mm}$ orbital shaking, further $20 \mathrm{sec}$ without shaking, and measurement of absorbance at $600 \mathrm{~nm}$. OD values measured in the plate reader were calibrated to represent the OD measurements of standard cuvette, which were found to correlate according to $\mathrm{OD}_{\text {cuvette }}=\mathrm{OD}_{\text {plate }} / 0.23$.

\section{Carbon labeling within proteogenic alanine and valine}

Mass isotopologues distributions (MID) can supply information regarding the topology and the fluxes in a metabolic network. By measuring the MID of specific amino acids, it is possible to infer the labeling pattern in 
precursors metabolites upstream to it in the biosynthesis pathway. For intermediates directly converted to amino acids, hydrolyzed protein samples hold several advantages such as increased throughput and sample stability. For MID analysis of hydrolyzed amino acids cells were cultured in $50 \mathrm{ml} \mathrm{M9} \mathrm{media} \mathrm{under} \mathrm{anaerobic} \mathrm{conditions} \mathrm{as}$ described above. M9 media used contained $0.2 \%$ unlabeled formate or ${ }^{13} \mathrm{C}$ labeled formate (Sodium formate- ${ }^{13} \mathrm{C}$, Sigma Aldrich 279412), and / or $0.2 \%$ unlabeled acetate or $1-{ }^{13} \mathrm{C}$-labeled acetate (Sodium acetate-1- ${ }^{13} \mathrm{C}$, SigmaAldrich 279293). After reaching stationary phase cells were harvested by centrifugation for $3 \mathrm{~min}$ at $5000 \mathrm{~g}$. Protein biomass was hydrolyzed with $6 \mathrm{~N}$ hydrochloric acid using standard protocols. Hydrolyzed amino acids were separated using ultra performance liquid chromatography (Acquity - Waters, USA) on a C-8 column (Zorbax Eclipse XBD - Agilent, USA) at a flow rate of $0.6 \mathrm{~mL} / \mathrm{min}$ and eluted off the column using a hydrophobicity gradient. Buffers used were: A) $\mathrm{H}_{2} \mathrm{O}+0.1 \%$ formic acid and B) acetonitrile $+0.1 \%$ formic acid with the following gradient: $100 \%$ of A (0-3 min), $100 \%$ A to $100 \% \mathrm{~B}(3-9 \mathrm{~min}), 100 \% \mathrm{~B}(9-13 \mathrm{~min}), 100 \% \mathrm{~B}$ to $100 \%$ A (13-14 min), 100\% A (14-20 min). Overall run time was 20 minutes. The UPLC was coupled online to a triple quadrupole mass spectrometer (TQS - Waters, USA). Data was acquired using MassLynx v4.1 (Waters, USA). Optimization of ionization parameters and determination of retention times was performed by direct infusion of amino acid commercial standards (Sigma-Aldrich, USA). Argon was used as the collision gas with a flow rate of $0.22 \mathrm{~mL} / \mathrm{min}$. Cone voltage was $25 \mathrm{~V}$, the capillary was set to $3 \mathrm{kV}$, source temperature was $150^{\circ} \mathrm{C}$, desolvation temperature was $500^{\circ} \mathrm{C}$, desolvation gas flow was $700 \mathrm{~L} / \mathrm{min}$, source offset 50 , cone gas flow was 250 $\mathrm{L} / \mathrm{min}$ and collision energy was $14 \mathrm{eV}$. MIDs were detected using multiple reaction monitoring (MRM) with the known molecular masses and the neutral loss of carbonyl carbon as a daughter ion (either 47 or $46 \mathrm{~m} / \mathrm{z}$, labeled and unlabeled respectively). Data analysis was performed using TargetLynx (Waters, USA).

\section{References}

(1) Zelcbuch, L., Antonovsky, N., Bar-Even, A., Levin-Karp, A., Barenholz, U., Dayagi, M., Liebermeister, W., Flamholz, A., Noor, E., Amram, S., Brandis, A., Bareia, T., Yofe, I., Jubran, H., and Milo, R. Spanning high-dimensional expression space using ribosome-binding site combinatorics (2013) Nucleic Acids Res 41, e98.

(2) Heckman, K. L., and Pease, L. R. Gene splicing and mutagenesis by PCR-driven overlap extension (2007) Nature protocols 2, 924-932.

(3) Baba, T., Ara, T., Hasegawa, M., Takai, Y., Okumura, Y., Baba, M., Datsenko, K. A., Tomita, M., Wanner, B. L., and Mori, H. Construction of Escherichia coli K-12 in-frame, single-gene knockout mutants: the Keio collection (2006) Mol Syst Biol 2, 2006-2008. 